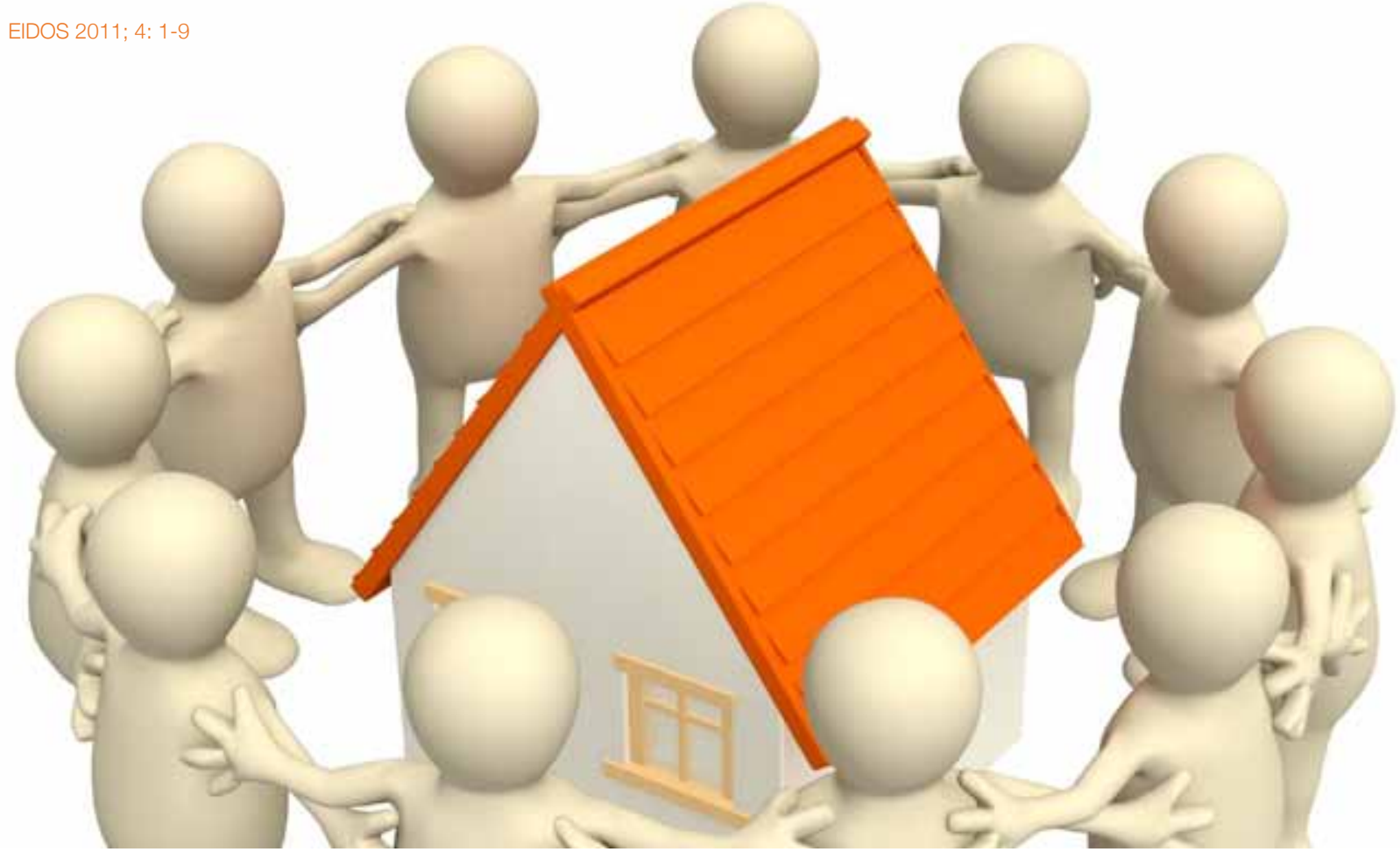

EIDOS, 4

Marzo-agosto 2011, 92-100

ISSN:1390-499X

elSSN:1390-5007

\title{
LA RESPONSABILIDAD SOCIAL COMO MODELO DE GESTIÓN EMPRESARIAL
}

Recepción/Received: 2011-04-20

Aceptación/Accepted: 2011-07-11

Publicado/Published: 2011-08-05

Resumen -La Responsabilidad Social Empresarial (RSE) es el compromiso voluntario asumido por la empresa de contribuir al desarrollo social sustentable, la misma que debe ser concebida desde un enfoque sistémico, instrumento integrador que considera diversos elementos y relaciones de la estructura de una organización, para la identificación de necesidades, determinación de estrategias, la selección, aplicación y evaluación de los resultados obtenidos, lo que genera el modelo de gestión que permite a la empresa incorporar dentro de su planificación estratégica herramientas para responder a las necesidades del entorno y sus stakeholders o grupos de interés los que deben ser involucrados en los procesos de toma de decisiones institucionales, estas acciones hacen que la organización alcance la excelencia.

\section{Palabras clave -Responsabilidad social, sostenibilidad, grupos de interés.}

Abstract -Social Business Responsibility (SBR) is a voluntary commitment assumed by the company to contribute social sustainable development; this responsibility must be conceived from a systematic approach, this is an integrating instrument which considers many elements and relations on the organization structure to identify needs, determine strategies, select, apply and assess the results gotten, which generates the appropriate management model that permits the company to add, as part of its strategic planning, some tools to meet necessities from stakeholders, interested groups and society; elements that must be involved in the process of making institutional decisions, such actions makes the organization reach excellence.

Keywords -Corporate Social Responsibility, stakeholders, sustainable.

1 Jorge Viteri Moya, jviteri@ute.edu.ec. Decano de la Facultad de Ciencias de la Ingeniería, Universidad Tecnológica Equinoccial.

2 María Belén Jácome, jvmb2304@ute.edu.ec. Coordinadora de Vinculación con la Colectividad de la Facultad de Ciencias de la Ingeniería, Universidad Tecnológica Equinoccial, 


\section{INTRODUCCIÓN}

En el actual escenario mundial, los principales desafíos para las organizaciones provienen de los vertiginosos cambios del entorno: la globalización, la competencia, la tecnología y el conocimiento, demandan a las empresas serias modificaciones en sus estructuras y estrategias [1], para cambiar las competencias y el compromiso ético de las empresas con la sociedad, enmarcado en un nuevo enfoque de gestión que relacione la Responsabilidad Social y el desarrollo sostenible [2], se considera que existe un contrato social implícito entre la sociedad y la empresa: la empresa tiene obligaciones con respecto a la sociedad y tiene que elaborar su política con la visión de rendir cuentas ante la sociedad de sus actuaciones [3]

El desarrollo sostenible, perdurable o sustentable, surge como respuesta al deterioro del medio ambiente ocasionado por un sistema económico mundial gobernado por las fuerzas del mercado [4], este término fue formalizado por primera vez en el Informe Brundtland en 1987, como "Satisfacer las necesidades de las generaciones presentes sin comprometer las posibilidades de las del futuro para atender sus propias necesidades", fruto de los trabajos de la Comisión Mundial de Medio Ambiente y Desarrollo de la Organización de las Naciones Unidas [5]. Dicha definición es ratificada en el Principio tres de la Declaración de Río (1992): "El derecho al desarrollo debe ejercerse en forma tal que responda equitativamente a las necesidades de desarrollo y ambientales de las generaciones presentes y futuras" [6], [7]. La sostenibilidad es un reto multidimensional que la empresa debe asumir hoy con una visión del mañana, como una estrategia empresarial que aborde asuntos actuales de reducción de costos, riesgos y cuidado medioambiental y los convierta en iniciativas empresariales y tecnológicas transformadoras que no sólo las lleve a innovar sino que alcancen el crecimiento y rentabilidad por una largo tiempo para la creación de "valor sostenible". [8]

El desafío que las organizaciones y la humanidad en general tienen ante estas declaraciones, es el de construir un nuevo modelo de desarrollo que permita satisfacer la calidad de vida de la población y conservar el medio ambiente para que las futuras generaciones dispongan de las mismas oportunidades [9], aunque el desafío mayor está en la implementación de un modelo de gestión empresarial que incorpore a la RS, el mismo que demanda de las empresas una administración integrada de los impactos: sociales - justos, económicos - viables y

11 Jorge Viteri Moya, Decano de la Facultad de Ciencias de la Ingeniería de la Universidad Tecnológica Equinoccial, jviteri@ute.edu.ec. ambientales - sanos de manera equilibrada, lo que se conoce como "The Triple Bottom Line"1 [10].

FUNDAMENTOS Y PRINCIPIOS

DE LA RESPONSABILIDAD SOCIAL

EMPRESARIAL COMO MODELO DE GESTIÓN

La responsabilidad social practicada por las empresas, ha sido definida por numerosos autores, organismos e institutos, algunas de estas se exponen a continuación:

- Compromiso de la empresa de contribuir al desarrollo económico sostenible, al trabajar con los empleados, sus familias, la comunidad local y la sociedad y en general para mejorar su calidad de vida. [11]

- Integración voluntaria, por arte de las empresas, de las preocupaciones sociales y medioambientales en sus operaciones comerciales y sus relaciones con sus interlocutores. [12]

- Relación que la empresa establece con todos sus públicos, a corto y a largo plazo, trasciende aquello que la empresa debe hacer por obligación legal. [13]

- Iniciativa generalmente de naturaleza voluntaria y se refiere a actividades que superan el mero cumplimiento de la legislación, cómo las empresas toman en consideración las repercusiones que sus actividades tienen sobre la sociedad. [14]

- Nueva forma de hacer negocios, en la que la empresa gestiona sus operaciones de forma sustentable en lo económico, social y ambiental, a la vez que reconoce los intereses de distintos públicos con los que se relaciona. [15]

- Compromiso voluntario que las organizaciones asumen frente a las expectativas concertadas que en materia de desarrollo humano integral se generan con las partes interesadas y que, partiendo del cumplimiento de las disposiciones legales, le permite a las organizaciones asegurar el crecimiento económico, el desarrollo social y el equilibrio ecológico. [16]

- Responsabilidad de una organización por los impactos de sus decisiones y actividades en la sociedad y en el ambiente a través de un comportamiento ético y transparente. [17]

- Operar una empresa de manera que rinde cuentas de los impactos sociales y medioambientales que esta crea por sus actividades, es un compromiso para desarrollar políticas que integren prácticas responsables dentro de las operaciones diarias de la empresa. [18]

2 Rendición de cuentas. Organización internacional sin ánimo de lucro que trabaja con socios en el mundo de los negocios, en el sector público y en la sociedad civil. 
- Producir bienes y servicios que la sociedad demanda, pero esos bienes y servicios deben ser responsables, que cumplan su función, que satisfagan las necesidades de la sociedad y no solo de las empresas, que hagan lo que dicen que hacen, que no produzcan daño a las personas y al medio ambiente, que hayan sido producidos con prácticas responsables con los trabajadores y con insumos no dañinos. [19]

Resultado del análisis de las definiciones de RSE se puede plantear: Responsabilidad social empresarial es el compromiso de la empresa para identificar los problemas que afecten a sus grupos de interés como: medio ambiente, pobreza, desigualdad de ingresos, atención de salud, hambre, desnutrición y analfabetismo, y emprender acciones que generen impactos sociales, económicos y ambientales positivos, con el aporte de soluciones basadas en la transparencia, pluralidad, sustentabilidad y ética; la meta es el desarrollo sustentable del ser humano y su entorno.

La responsabilidad social empresarial es un concepto dinámico y multidimensional, de las definiciones analizadas se identifica los elementos en común: conjunto de prácticas obligatorias y voluntarias; contribución al desarrollo social, crecimiento económico y preservación del medio ambiente es decir la Triple cuenta de resultados para contribuir al desarrollo sostenible de la sociedad; integración y respuesta de los problemas sociales y medio ambientales del entorno donde opera e incorporación de los grupos de interés a la toma de decisiones.

En la actualidad, la RS plantea nuevos escenarios y desafíos, la incorporación de esta como un modelo sistémico, que aborde la planificación estratégica de la organización y le permita alcanzar la excelencia. En relación a estos retos, la ONG Británica Accountability realizó en el año 2007 un estudio del progreso del Estado de la Competitividad Responsable con alcance global, evaluó las prácticas empresariales responsables

3 Conjunto de leyes fundamentales que fijan la organización política del Estado y establece los derechos y obligaciones básicas de ciudadanos y gobernantes. En forma documental, consta de nueve títulos a su vez, conformados por capítulos y estos divididos en secciones que, finalmente, se componen de artículos. (Asamblea Constituyente, 2008)

4 Buen vivir.

5 Ética con los Trabajadores, Ética con la Comunidad, Ética con el Gobierno y Ética con el Medio Ambiente.

6 Organización privada, sin fines de lucro, formada por un equipo de personas con positiva y vasta experiencia en el campo empresarial, en la academia, en la cátedra, en múltiples responsabilidades públicas y privadas. en 108 países y emitió un informe denominado "El Índice de Competitividad Responsable" (ICR), que determina los países que tienen condiciones sociales y están desarrollando políticas públicas para fomentar la competitividad responsable, según este informe el Ecuador está en la posición 79, en un ranking de 108 países [20], estos datos reflejan la realidad que vivía el país en este período, sin embargo cabe señalar que a partir del 2007 se realizan cambios trascendentales en las políticas públicas y lineamientos que rigen al Ecuador, en los que se incluyen conceptos de RS y sustentabilidad, entre los más importantes se pueden mencionar los siguientes: La Constitución Ecuatoriana ${ }^{3}$ aprobada en referéndum en septiembre de 2008, con sus 44 artículos, establece una nueva visión en la que el centro del desarrollo es el ser humano y el objetivo final es el alcanzar el sumak kawsay ${ }^{4}$ y la estructuración del Plan Nacional para el Buen Vivir 2009-2013.

Además del cambio establecido en la constitución, el gobierno nacional a través del Programa CreEcuador del Ministerio de Coordinación de la Producción, Empleo y Competitividad, impulsa los Sellos Hace Bien y Hace Mejor, como una iniciativa que pretende reconocer a las empresas ecuatorianas comprometidas con el cumplimiento de las cuatro Éticas Empresariales ${ }^{5}$ [21]. Otras organizaciones no gubernamentales fomentan la incorporación de prácticas de RS, dentro de las organizaciones, como: el Consorcio Ecuatoriano para la Responsabilidad Social (Ceres), es el primer organismo dedicado exclusivamente al tratamiento de los temas de Responsabilidad Social en el país [22], en el año 2005 pasa a formar parte de la Red Continental de Forum Empresa, el Instituto Ecuatoriano de Responsabilidad Social Empresarial $(\mathrm{RSSE})^{6}$, la Plataforma de Responsabilidad Social que es una coalición de organizaciones de la Sociedad Civil Ecuatoriana que tienen como fin contribuir al ejercicio de la Responsabilidad Social en las instancias públicas y privadas del Ecuador.y a la cual pertenece la Universidad Tecnológica Equinoccial.

La empresa privada ecuatoriana también realiza varias actividades para la incorporación de la RSE como un modelo de

8 Normas internacionales que promueve la adopción de un enfoque basado en procesos cuando se desarrolla, implementa y mejora la eficacia de un sistema de gestión de la calidad. El modelo de un sistema de gestión de la calidad basado en procesos, aplica la metodología conocida como "Planificar - Hacer - Verificar - Actuar". (Organización Internacional de Normalización, 2008)

9 Fundación Europea para la Gestión de la Calidad

10 Los conceptos fundamentales del Modelo son: la orientación hacia los resultados y hacia el cliente, el liderazgo y la constancia, la gestión por procesos y hechos, el desarrollo y la implicación de las personas, el aprendizaje y la mejora continua, la colaboración, y la responsabilidad social . (European Foundation for Quality Management, 2004)

7 Asociación civil fundada en diciembre de 1988. 
Cuadro 1: Normas de gestión de la RSE.

\begin{tabular}{|c|c|c|c|}
\hline Norma & Organización & Alcance & Descripción \\
\hline SA $8000-2008$ & $\frac{\text { Social Accountability }}{\text { International (SAI). }}$ & Norma auditable y certificable. & $\begin{array}{l}\text { Promueve la implantación de los derechos humanos y } \\
\text { laborales. Está enfocada en nueve áreas esenciales: trabajo } \\
\text { infantil, trabajo forzado, salud y seguridad, libertad de } \\
\text { asociación, discriminación, medidas disciplinarias, horario } \\
\text { de trabajo, remuneración, implementación eficiente de un } \\
\text { sistema de gestión y revisión. }\end{array}$ \\
\hline SGE21:2008 & Foroética & Norma certificable. & $\begin{array}{l}\text { Busca establecer, implantar y evaluar la Gestión Ética y } \\
\text { Socialmente Responsable en las organizaciones. Tiene seis } \\
\text { capítulos y está estructurada en nueve áreas de gestión: alta } \\
\text { dirección, clientes, proveedores, personas que trabajan para } \\
\text { la organización, entorno social y ambiental, inversores, } \\
\text { competencia, administraciones públicas. }\end{array}$ \\
\hline ISO 26000:2010 & $\begin{array}{l}\text { Organización } \\
\text { Internacional de } \\
\text { Normalización }\end{array}$ & Norma no certificable. & $\begin{array}{l}\text { Proporciona las directrices para establecer, implementar, } \\
\text { mantener y mejorar la RSE. Estructurada sobre la base de } \\
\text { siete principios de RSE: rendición de cuentas, transparencia, } \\
\text { comportamiento ético, respeto por los intereses de los } \\
\text { stakeholders, respeto por la Ley, respeto por las normativas } \\
\text { internacionales de comportamiento y respeto por los } \\
\text { derechos humanos. }\end{array}$ \\
\hline AA1000: 1999 & $\begin{array}{l}\text { Institute Of Social } \\
\quad \text { And Ethical } \\
\text { Accountability }\end{array}$ & $\begin{array}{l}\text { Es un estándar de aplicación } \\
\text { general para evaluar, atestiguar y } \\
\text { fortalecer la credibilidad y } \\
\text { calidad del informe de } \\
\text { sostenibilidad de una } \\
\text { organización. }\end{array}$ & $\begin{array}{l}\text { Su aplicación está basada en un compromiso de } \\
\text { responsabilidad la inclusión de los stakeholders en la toma } \\
\text { de decisiones y los tres principios: relevancia, exhaustividad } \\
\text { y capacidad de respuesta. }\end{array}$ \\
\hline ISO 9001:2008 & $\begin{array}{l}\text { Organización } \\
\text { Internacional de } \\
\text { Normalización }\end{array}$ & Norma internacional certificable. & $\begin{array}{l}\text { Promueve la adopción de un enfoque basado en procesos } \\
\text { cuando se desarrolla, implementa y mejora la eficacia de un } \\
\text { sistema de gestión de la calidad, para aumentar la } \\
\text { satisfacción del cliente mediante el cumplimiento de sus } \\
\text { requisitos. Aplica la metodología conocida como: Planificar, } \\
\text { Hacer, Verificar y Actuar. }\end{array}$ \\
\hline ISO 1400: 2004 & $\begin{array}{l}\text { Organización } \\
\text { Internacional de } \\
\text { Normalización }\end{array}$ & Norma internacional certificable. & $\begin{array}{l}\text { Estable la incorporación de un sistema de gestión ambiental. } \\
\text { Especifica los requisitos para un sistema de gestión } \\
\text { ambiental que le permita a una organización desarrollar e } \\
\text { implementar una política y unos objetivos que tengan en } \\
\text { cuenta los requisitos legales y la información sobre los } \\
\text { aspectos ambientales significativos. }\end{array}$ \\
\hline OHSAS 18.001: 2007 & $\begin{array}{c}\text { OHSAS }^{12} \\
\text { Proyect Group }\end{array}$ & Norma internacional certificable. & $\begin{array}{l}\text { Estándares voluntarios internacionales relacionados con la } \\
\text { gestión de seguridad y salud ocupacional, sistema que } \\
\text { entrega requisitos para implementar un sistema de gestión de } \\
\text { salud y seguridad ocupacional, habilitando a una empresa } \\
\text { para formular una política y objetivos específicos asociados } \\
\text { al tema, considerando requisitos legales e información sobre } \\
\text { los riesgos inherentes a su actividad. }\end{array}$ \\
\hline NBR 16001:2004 & $\begin{array}{l}\text { Asociación Brasileña } \\
\text { de Normas Técnicas } \\
\text { (ABNT) }\end{array}$ & Norma auditable y certificable. & $\begin{array}{l}\text { Se basa en la mejora metodología de mejora continua. Los } \\
\text { principios básicos de la norma son: buenas prácticas de } \\
\text { gobernabilidad, combate a la piratería, fraude y corrupción, } \\
\text { prácticas leales de competencia, derechos del niño y del } \\
\text { adolescente, derechos del trabajador promoción de la } \\
\text { diversidad y lucha contra la discriminación, compromiso con } \\
\text { el desempeño profesional, promoción de la salud y } \\
\text { seguridad, protección del medio ambiente y procedimientos } \\
\text { sustentables de producción y acciones sociales de interés } \\
\text { público. }\end{array}$ \\
\hline EMAS: 1993 & Unión Europea & Norma auditable y certificable. & $\begin{array}{l}\text { El Sistema Comunitario de Gestión y Auditoría } \\
\text { Medioambientales que permite: Evaluar y mejorar su } \\
\text { comportamiento y medio ambiental, además de difundir la } \\
\text { información pertinente relacionada con su gestión } \\
\text { medioambiental, al público y a otras partes interesadas. }\end{array}$ \\
\hline
\end{tabular}


gestión, así importantes organizaciones ecuatorianas, alcanzaron el distintivo, "Empresa Ejemplar Socialmente Responsable" en América Latina, otorgado por el Centro Mexicano para la Filantropía (CEMEFI), Forum empresa y Alianza para la Responsabilidad Empresarial en México (AliaRSE) [23] [24].

Las empresas ecuatorianas se encuentran en una etapa de transición, en el rol que desempeñan dentro de la sociedad, al pasar de prácticas de ayuda social como beneficencia a la RSE, así se encamina los primeros pasos para incluir a la RSE en su gestión.

Para cumplir con este desafío, las empresas tienen que implementar, un modelo sistémico de gestión para la incorporación de la RS, que permita la planificación, implementación, seguimiento y evaluación de los resultados, metodología abordada por los diversos modelos, enfoques o tendencias que denotan la excelencia empresarial, entre ellos: las normas propuestas por la Organización Internacional para la Estandarización ISO $^{8}$ sobre calidad, ambiente, gestión ambiental, gestión de la seguridad industrial y responsabilidad social; el modelo desarrollado por la European Foundatión for Quality Management de excelencia empresarial y las normas estructuradas por organizaciones internacionales para la gestión de la RSE AA1000, SGE21:2008, SA 8000 - 2008 [25], [26], [27], [28], [29], [30].

Las normas Las organizaciones responsable de la creación, alcance y una breve descripción se resumen en el Cuadro 1.

Cabe indicar que estas normas para la incorporación de la RSE se fundamentan en el Ciclo de Deming. [31]

El ciclo de Deming es una metodología estándar y universalmente aceptada en las normas de gestión de la calidad[32], permite adoptar el enfoque sistémico[33], como un instrumento integrador que considera diversos elementos y relaciones de la estructura de una organización, para la identificación de necesidades, determinación de estrategias, la selección, aplicación y evaluación de los resultados obtenidos [34] [35] [36] [37], lo expuesto justifica la aplicación del ciclo de Deming, como una herramienta de gestión para la incorporación de la RS dentro de las organizaciones.

La aplicación de la Responsabilidad Social dentro de la planificación estratégica de las organizaciones se puede definir como un modelo de gestión que integra el respeto al ambiente, el ejercicio de los derechos humanos y derechos de los trabajadores y el compromiso con los grupos de interés; que va desde la planeación, hasta la implementación, seguimiento y evaluación; que genera un proceso de mejora continua y crea valor para los stakeholders(ver figura 1) y tiene su origen en una cultura organizacional basada en principios y valores que crea valor no solo para la institución sino también para los stakeholders a fin de garantizar en el largo plazo la sostenibilidad y consistencia de la organización; esto permite la construcción de la imagen institucional, entendida como aquello que el entorno percibe, es la identidad observada y valorada.

La implementación de la RS como un modelo de gestión empresarial, consta de cuatro etapas aplicadas en el orden siguiente: planificación, implementación de medidas, control y seguimiento y evaluación, las que se describen a continuación.

\section{Etapa 1 - Planificación.}

En esta etapa, se realiza: análisis de la situación interna y externa, estructuración de la misión y visión, la definición de objetivos estratégicos, establecimiento del plan de acción y la asignación de recursos [38]

La planificación permite a la empresa: recolectar información e ideas, para la toma de decisiones y construcción de consensos entre los diferentes puntos de vista de los grupos de interés, para obtener credibilidad, apoyo, construir una imagen aceptada por la sociedad; estructurar el camino a seguir, basado en el estado actual y con una visión o estado que se pretende alcanzar después de la implementación.

\section{a. Análisis de la situación interna y externa}

El análisis de la situación interna y externa en la que se desenvuelve la organización, permite identificar las oportunidades y amenazas, para este estudio se debe considerar a los "Stakeholders" o grupos de interés, estos se refieren a la persona, grupo de personas u organización que pueden estar directa o indirectamente relacionadas con la institución, debido a que las acciones, objetivos o politicas organizacionales podrían afectarlas [39]. Las organizaciones pueden tener varios tipos de stakeholders cada cual con diferente nivel de involucración, compromiso o influencia y muchas veces con intereses diferentes y en conflicto, a los que se los puede clasificar en [40]:

Público Interno: son los que forman parte de la estructura interna de la empresa (accionistas o directivos; trabajadores)

Público Externo: están relacionados indirectamente con la empresa (clientes, consumidores, proveedores, la comunidad, el medio ambiente)

Para el análisis de la realidad interna y externa de la organización, se aplica la técnica FODA, que permite identificar y evaluar los factores positivos (fortalezas y oportunidades) y negativos (debilidades y amenazas) para adoptar decisiones 
sobre objetivos, cursos de acción y asignación de recursos sustentados en este análisis.

Para realizar este análisis, existen dos caminos reactivamente, o de forma proactiva, está última busca anticiparse a las demandas de la sociedad, para lo que es necesario mantener un dialogo en doble sentido, gestionado a priori y no posteriori, la comunicación de empresa es estructurada en el momento de su constitución de tal manera que se armonizan todas las acciones que se van a llevar a cabo antes de que la necesidad se genere, para superar las tres generaciones de relaciones empresariales con los stakeholders [41]:

- Relación forzada, bajo presión, realizada sin ninguna planificación, el objetivo es dar solución a problemas a través de la concesión de beneficios localizados, para mitigar de esta forma el impacto impositivo a la imagen empresarial.

- Relación sistemática, de influencia mutua en la toma de decisiones, existe una mayor comprensión de las necesidades de los stakeholders.

- Relación integral, proactiva, plasmada en la Planificación estratégica empresarial, logrando la competitividad sostenible.

\section{b. Estructuración de la misión y visión}

La misión y visión son dos elementos claves de la planificación estratégica, permiten a las instituciones ubicarse y enfocar sus acciones en congruencia con la filosofía de la organización [42].

Al precisar la visión y misión de las instituciones se establece el marco de referencia dentro del cual se incorpora la responsabilidad social al ADN de la organización como una filosofía de trabajo, que supere los tres niveles de la Responsabilidad Social Empresarial (RSE): (1) Voluntaria, que se da cuando existe el deseo de incorporar a la RS, pero sin un fin claro, etapa que se debe superar. (2) Implementación, etapa que integra los conceptos de RS al actuar de la organización. (3) Transición, etapa en la que se logra la transformación, incorpora a la RS en todas las áreas de gestión; es vital que los stakeholders sean parte de este proceso, para garantizar que éste refleje sus intereses y en consecuencia se sientan parte del compromiso de la institución.

La misión y la visión estructuradas con RS, están estrechamente relacionadas con los valores éticos de las organizaciones, que deben comunicar los compromisos de la empresa y reflejar la forma de cómo se relaciona con los grupos de interés, entre estos valores se destacan: la honestidad, la integridad, el respeto, la transparencia y la apertura [43].

\section{c. Definición de objetivos estratégicos}

La responsabilidad social debe estar dentro de la estrategia misma de la empresa y se debe alinear con las operaciones y procedimientos que ejecuta la organización en el día a día, así los objetivos estratégicos son la enunciación de lo que se desea conseguir para el logro de la misión, identifica los acuerdos que se deben consensuar con los grupos de interés y los resultados a lograr [44].

En la estructuración de los objetivos se debe establecer las estratégicas, indicadores y metas que pemitan su evaluación [45].

\section{d. Plan de acción}

Comprende el conjunto de estrategias, actividades, proyectos que se van a realizar aplicadas para el logro de los objetivos, deben ser coherentes con estos y manifestar de manera concreta y práctica la filosofía de la institución [46].

\section{Etapa 2 - Implementación}

Esta etapa comprende la aplicación de las acciones y medidas necesarias para ejecutar los procesos definidos en la planificación, con los recursos asignados y la delegación de tareas que permitan lograr con mayor eficacia la aplicación del plan e impulsen la consecución de los objetivos. [47].

La preparación para la implementación del plan, requiere que la empresa desarrolle mecanismos y sistemas para realizar el seguimiento y evaluación del plan, así como, diseños organizativos que apoyen las estrategias.

\section{Etapa 3 - Seguimiento}

El sistema de seguimiento verifica que se cumplan los objetivos o en algunos casos se reajusten para poder cumplirlos, incluye todas las actividades de gestión que tiene por objeto asegurar que los resultados actuales respondan a los resultados planeados, tiene los componentes que se examinan a continuación:

- Indicadores que relacionan entre sí los cursos de acción con el logro de los objetivos estratégicos contemplados en el plan.

- Recopilación de datos y el manejo de los registros de las actividades del plan, a fin de que los datos requeridos por los indicadores sean compatibles con las estadísticas existentes.

\section{Etapa 4 - Evaluación}

En esta etapa se estudian los resultados obtenidos en el seguimiento, para: modificar los procesos, dar soluciones a los problemas, identificar cambios y re-definir objetivos de mejora continua. Todas estas acciones forman parte del plan de mejora, que permite una gestión eficiente y eficaz en busca de la excelencia empresarial [48]. 


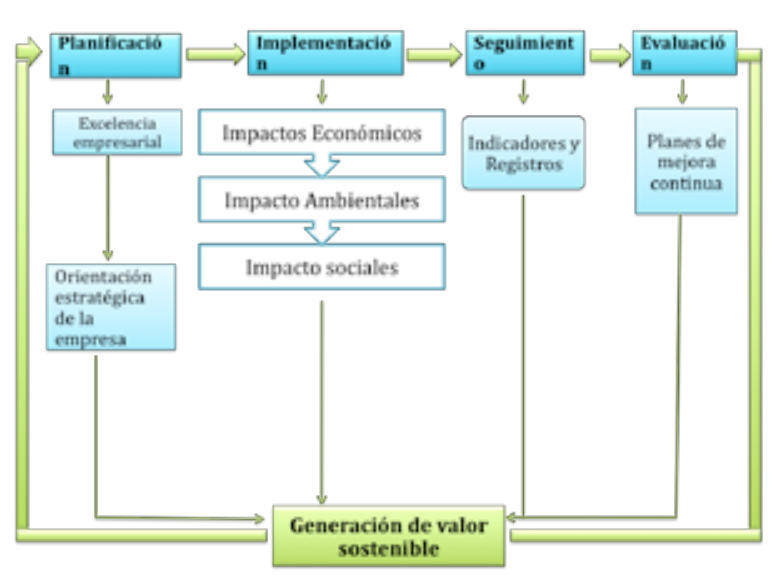

Figura 1. Modelo de Gestión para incorporar la Responsabilidad social Empresarial

\section{CONCLUSIONES}

La RSE es el compromiso de la empresa para identificar y aportar soluciones basadas en la transparencia, pluralidad, sustentabilidad y ética, a los problemas de: pobreza, contaminación del ambiente, desigualdad de ingresos, atención de salud, hambre, desnutrición y analfabetismo, que afectan a sus grupos de interés; para generar impactos sociales, económicos y ambientales positivos.

Las empresas ecuatorianas encaminan los primeros pasos para incluir a la RSE en su gestión, para superar el marketing social y actuar como organizaciones responsables con el desarrollo sustentable de sus stakeholders y del entorno. Además de algunas organizaciones no gubernamentales que fomentan la aplicación de prácticas de RS.

El ciclo de de Deming aplicado a la gestión de la RSE, es una herramienta que facilita la incorporación de indicadores para medir los impactos generados dentro de la estructura organizacional, además de constituirse en un sistema de mejora continua para planificar, hacer, verificar y actuar.

La RS como un modelo de gestión aplicado a las empresas permite cumplir con las normativas legales nacionales e internacionales, aunque este no se considera una obligatoriedad se constituye en la actualidad como una herramienta global necesaria para alcanzar la competitividad y sustentabilidad empresarial.

\section{BIBLIOGRAFÍA}

[1] Viteri Moya, J. R. (2007). La universidad y la responsabilidad social empresarial. Revista Campus UTE N 46, 10.

[2] Abascal Rojas, F. (2005). Marketing social y ética empresarial. Madrid: ESIC. Martínez H, H. (2005). La ética empresarial al comienzo del nuevo milenio. Facultad de ciencias económicas - Universidad Javeriana: Bogotá.

[3] Martínez H, H. (2005). La ética empresarial al comienzo del nuevo milenio. Facultad de ciencias económicas - Universidad Javeriana: Bogotá.

[4] Jacobs, M. (1996). La ecnomía verde: Medio ambinte, desarrollo sotenible y la política del futuro. Barcelona: ICARIA - FUHEM.

[5] Schmidheing, S. (1992). Chamging Course - a Global bussiness perspective on development and the environment. Massachussets: Institute of tecnnology Massachussets.

[6] Instituto Interamericano de Cooperación para la Agricultura. (1993). Agenda 21 - Desarrollo sostenible en Chile. San José de Costa Rica: Instituto Interamericano de Cooperación para la Agricultura .

[7] Sánchez Sánchez, H. (2008). Código de Derecho Internacional Ambiental . Bogotá: Universidad del Rosario.

[8] Senge, P. (2009). La revolución necesaria. Colombia: Grupo Editorial Norma

[9] Gómez Navarro, T. (2002). Ecología industrial. Producción industrial sostenible. In S. Capuz Rizo, T. Gómez Navarro, J. L. Vivancos Bono, R. Viñoles Cebolla, P. Ferrer Gisbert, R. López García, et al., Eco diseño. Ingeniería del ciclo de vida para el desarrollo de productos sostenibles (pp. 21-40). Valencia: Universidad Politécnica de Valencia.

[10] Blanco Cordero, M. (2004). Gestión ambiental. Camino al desarrollo sostenible. San José de Costa Rica: Editorial Universidad Estatal a Distancia.

[11]World Business Council for Sustainable Development. (1992). El Caso empresarial para el desarrollo sostenible. Estados Unidos de Norte América: World Business Council for Sustainable Development.

[12] Unión Europea. (2002). Libro verde la comisión Europea. Barcelona: ESADE - IPES.

[13] Instituto Ethos Brazil. (2005). Conceptos básicos e Indicadores de Resposabilidad Social Empresarial. Venezuela: Instituo Ethos y Cetro de divulgación del Conocimiento Económico "CEDICE".

[14] Organización Internacional del Trabajo. (2007). Responsabilidad Social de la Empresa. Panorama laboral 2007, 22 - 25. 
[15] Consorcio Ecuatoriano para la Responsabilidad Social - CERES. (2008). Responsabilidad Social Empresarial mis primeros pasos. Quito: Consorcio Ecuatoriano para la Responsabilidad Social - CERES.

[16] Instituto Colombiano de Normas Técnicas y Certificación. ICONTEC. (2008). Guía Técnica colombiana 180 de RS. Bogotá: ICONTEC.

[17] Organización Internacional de Normalización. (2010). Guidance on social responsibility. Suiza: Organización Internacional de Normalización .

[18] Kamauff, J. (2010). Manager's guide to operations management. USA. McGrawHill.

[19] Vives, A. (2011) La responsabilidad Social de la empresa ante la Sociedad. USA. Banco Interamericano de Desarrollo.

[20] Zadey, S., \& MacGillivray, A. (2007). El estado de la competitividad responsable. Inglaterra: Accountability.

[21] Idrovo Andrade, I. (2010). Guía de autoevaluación para el sello HACE MEJOR. Ecuador: Ministerio de Coordinación de la Producción, Empleo y Competitividad.

[22] Galán Melo, A. R. (2008). Evaluación crítica de los discursos de Responsabilidad Social Coorporativa. Quito: Universidad Andina simón Bolivar - Sede Ecuador.

[23] Gonzalez Tirado, A. (17 de Marzo de 2010). Tercer Encuentro Latinoamericano de Empresas Socialmente Responsables. Recuperado el 7 de Abril de 2011, de Circulo Verde: www. circuloverde.com.mx/es/cont/rss/Tercer_Encuentro_Latinoamericano_de_Empresas_Socialmente_Responsables.shtml

[24] Diners Club del Ecuador. (2009). Informe de Responsabilidad Corporativa Diners Culb del Ecuador 2009. Quito: Diners Club del Ecuador.

[25] Institute of Social and Ethical Accountability. (1999). Accountability 1000 (AA1000). Londres : Institute of Social and Ethical Accountability.

[26] Social Accountability International. (2008). Social accountability 8000. Nueva York: SAl.

[27] Foro para la Evaluación de la Gestión Ética. (2008). Norma para la Evaluación de la Gestión Ética y Socialmente Responsable en las organizaciones (SGE 21:2008). Madrid: Forética.

[28] Organización Internacional de Normalización. (2008). Sistemas de gestión de la calidad - Requisitos. Ginebra: ISO.

[29] Fundación más Familia. (2010). EFR - Modelo de Gestión. Retrieved Febrero 10, 2011, from http://www.certificadoefr. org/index. php?section=modelodegestion.
[30] Viteri Moya, J. (2010). Responsabilidad Social. Quito: Equinoccio Series Académicas N 07 - UTE.

[31] Olcese, A., Rodríguez, M. Á., \& Alfaro, J. (2008). Manual de la empresa responsable y sostenible. Madrid: McGraw - Hill .

[32] Segarra, O. (2007). Los 15 círculos de autoliderazgo. BarceIona: Gestión 2000.

[33] Izar Landeta, J. M., \& Gonzáles Ortiz, J. H. (2004). Las 7 herramientas básicas de la calidad. San Luis Potosi: Universidad autónoma de San Luis Potosi.

[34] Lépiz Jiménez, C. (2003). La administración y planificación como procesos. San José de Costa Rica: Universidad Estal a Distancia San José de Costa Rica.

[35] Valdés Hernandez, L. A. (2005). Planeación estratégica como enfoque sistémico. México: Universidad Autónoma de México.

[36] O'Connor, J., \& McDermott, I. (2007). Introducción al Pensamiento sistémico. Barcelona: Urano.

[37] Senger, P. (2008). La quinta disciplina: el arte y la práctica de la organización abierta al aprendizaje. Buenos Aires: Granica.

[38] Riveros Silva, P. E. (2007). Sistema de Gestión de la calidad del servicio. Bogotá: Ecoe Ediciones.

[39] Argondoña, A. (1998). La Teoría de los Stakeholders y el Bien común. España: IESE Business Scholl - Universidad de Navarra.

[40] Accountability, United Nations Environment Programmer, Stakeholder Research Associates Canada Inc. (2006). el Compromiso de los stakeholders. Manual para la práctica de las realciones con los grupos de interés. Canada: Accountability.

[41] Carrillo Durán, M. V., Núñez de Prado Clavell, S., Delgado Pérez, J. P., \& Castillo Díaz, A. (2009). Comunicación Integral y Responsabilidad Social de las organizaciones. MéxicO: PEARSON.

[42] Secretaria de Educación Pública de los Estados Unidos Mexicanos. (2002). Planeación Institucional Metodología. México: Secretaria de Educación Pública de los Estados Unidos Mexicanos.

[43] Correa, E., Flynn, S., \& Amit, A. (2004). Responsabilidad Social Corporativa en América Latoma: una visión empresarial. Santiago de Chile: Organización de las Naciones Unidas (ONU).

[44] Torresano, M. (2008). La responsabilidad social empresarial del concepto a la práctica. Revista Económica del IDE - Perspectiva , 2 - 5. 
[45] Delgado Hernández, D. J. (2008). Planeación estratégica como base para la implementación de un sistema de gestión tecnológica en el contexto mexicano. México: ALAS.

[46] Udaondo Durán, M. (1991). Gestión de la Calidad. Madrid: Díaz de Santos S.A.

[47] Molins Pera, M. (1998). Teoría de la Planificación. Caracas: Facultad de Humaniddes y Educación - Universidad Central de Venezuela.

[48] Membrado Martínez, J. (2007). Metodologías avanzadas para la planificación y mejora. España: Díaz de Santos.

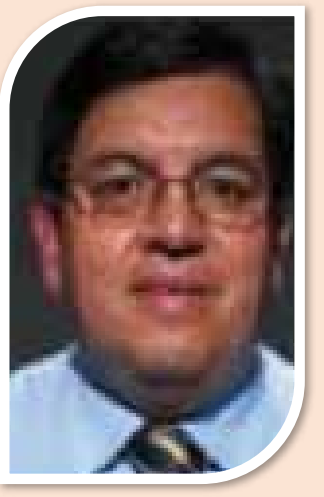

Jorge Rene Viteri Moya

Máster en Administración de Empresas, Magíster en Gestión de la Producción y Máster en Pedagogía Profesional. Tiene la Certificación Black Belt Six Sigma del Managment \& Technology member of APICS The Association for operations Managment, es Auditor Interno de las Normas ISO 9001. Se ha desempeñado como Gerente de Producción de EDESA - Quito y como Gerente Técnico Corporativo de EDESA-VENCERAMICA, Venezuela. Es Presidente de la Comisión Académica de la Asociación Ecuatoriana de Instituciones de Enseñanza de Ingeniería - ASECEl; es Representante de la Universidad Tecnológica Equinoccial ante la Plataforma de Responsabilidad Social - FLACSO; es Representante Delegado Principal del CONESUP ante el Observatorio Ambiental del DistritoMetropolitano de Quito; es Miembro del Comité Consultivo de Certificación S.G.S. del Ecuador. En la actualidad se desempeña como Decano de la Facultad de Ciencias de la Ingeniería de la Universidad Tecnológica Equinoccial. 


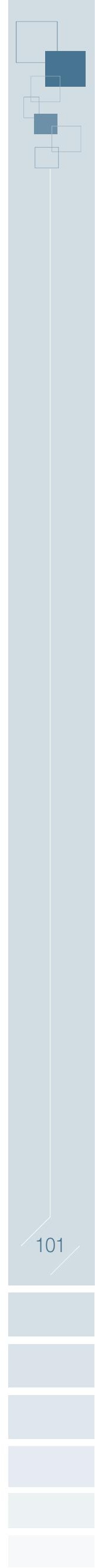




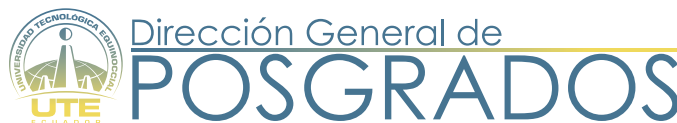

Dirección General de Posgrados

Bourgeois N34-102 y Rumipamba, Bloque B, tercer piso

Telfs: (02) 2990800 / (02) 2446290 / (02) 2446258

Ext.: 2431, 2412, 2417 / posgrado@ute.edu.ec

\section{Campus Santo Domingo de los Tsáchilas}

Teléfonos: (02) 3753108 / 093095309

E-mail:Imartinez@ute.edu.ec

\section{Campus Península de Santa Elena}

Teléfonos: (04) $2930051 /$

(04) 2776696 / (04) 2777696

Email:pmolina@ute.edu.ec

\section{Oficina Manta:}

Teléfono: (05) 2612828

E-mail: rjijon@ute.edu.ec

Oficina Cuenca:

Teléfono: (07) 2882216

E-mail: mjaramillo@ute.edu.ec

Oficina Ambato:

Teléfono: (03) 2822453

E-mail: hgallegos@ute.edu.ec

Oficina Guayaquil:

Teléfonos: (04) 2631008 / (04) 2631009

E-mail: iarmijos@ute.edu.ec

\section{Oficina lbarra:}

Teléfono: (06) 2600980

E-mail:jbaez@ute.edu.ec

\section{Oficina Loja:}

Teléfono: (07) 2550200

E-mail: rotorres@ute.edu.ec

Oficina Riobamba:

Teléfono: (03) 2951186

E-mail: nbrito@ute.edu.ec 